\title{
Foreword
}

Most of the information in Astrophysics is based on the analyse of the light emitted by astronomical objects such as stars, galaxies, planetes, or giant molecular clouds. In order to understand what are the physical conditions in these very different objects we must follow the "history" of the photons from their emission, sometime million of parsecs from us, to their capture. This light analyse is very rich since photons are keeping "memory" of the various thermodynamical conditions they were flowing out, from very dense regions deep into stellar atmospheres to dusty environment or the relative vacuum of the interstellar medium. This analyse is based on the radiative transfer theory which is partially the scope of the present proceedings.

This book presents the text of the lectures given during the second GRETA (GRoupE Transfert en Astrophysique) meeting in November 2001 at the Institut d'Astrophysique de Paris (IAP). The GRETA is a group of more than 40 researchers and doctoral or post-doctoral students from 13 different laboratories working in the field of radiative transfer. More details about the GRETA activities can be found at the URL: http://www.obsnice.fr/stee/transfert/transfert.html

This second meeting was more focused on the radiative transfer coupling with hydrodynamics and we hope the reader will find these proceedings useful since it is more or less a complete review of the radiative transfer activities lead in France and at Heidelberg University (Germany).

This work has been supported by the Programme national de Physique Stellaire (PNPS) which is part of the INSU division and the Comité Doyen Lépine (Ville de Nice).

\section{Philippe Stee}

(C) EAS, EDP Sciences 2002

DOI: $10.1051 /$ eas/20020501 